\title{
Synthesis of Copper Nanoparticles Coated with Nitrogen Ligands
}

\section{Rubén Sierra-Ávila, Marissa Pérez-Alvarez, Gregorio Cadenas-Pliego, Carlos Alberto Ávila-Orta, Rebeca Betancourt-Galindo, Enrique Jiménez-Regalado, Rosa Martha Jiménez-Barrera, and Juan Guillermo Martínez-Colunga}

Centro de Investigación en Química Aplicada, 25294 Saltillo Coahuila, Mexico

Correspondence should be addressed to Marissa Pérez-Alvarez; marisa.perez@ciqa.edu.mx and Gregorio Cadenas-Pliego; gregorio.cadenas@ciqa.edu.mx

Received 18 January 2014; Accepted 28 April 2014; Published 28 May 2014

Academic Editor: Claude Estournès

Copyright (C) 2014 Rubén Sierra-Ávila et al. This is an open access article distributed under the Creative Commons Attribution License, which permits unrestricted use, distribution, and reproduction in any medium, provided the original work is properly cited.

\begin{abstract}
The synthesis of copper nanoparticles was studied by wet chemical methods using copper sulfate pentahydrate $\left(\mathrm{CuSO}_{4} \cdot 5 \mathrm{H}_{2} \mathrm{O}\right)$ and nitrogen ligands allylamine (AAm) and polyallylamine (PAAm) as stabilizers. The results suggest that the use of these ligands leads to the exclusive formation of metallic copper nanoparticles (Cu-NPs). The use of partially crosslinked polyallylamine (PAAmc) leads to nanoparticles (NPs) with low yields and high coating content, while linear PAAm leads to NPs with high yields and low coating content. The chemical composition of the particles was determined by XRD and average particle diameters were determined by the Debye-Scherrer equation. TGA analysis provided evidence of the content and thermal stability of the coating on the nanoparticles and PAAm. The morphology, particle size distribution, and presence of PAAm coating were observed through TEM. The use of AAm in the synthesis of NPs could be a good alternative to reduce costs. By using TGA, TEM, and DSC techniques, it was determined that synthesized NPs with AAm presented a coating with similar characteristics to NPs with PAAm, suggesting that AAm underwent polymerization during the synthesis.
\end{abstract}

\section{Introduction}

Since 1990, scientific and technological research on the synthesis of inorganic nanoparticles has increased markedly due to their interesting physical properties and potential applications $[1,2]$. This is mainly attributed because of their small size, shape, composition, and high surface area to volume ratio [3]. Particular attention has been given to metal particles, especially copper particles due to the fact that they exhibit unique physicochemical properties not seen in other materials and differ from their mass-counterparts in properties such as catalytic [4], optical [5], electrical [6], electronic [7], thermal [8], magnetic [9], and antimicrobial [10, 11]. Because of their attractive features nanoparticles of metallic copper can be used in different fields at laboratory- and industrial-scale production, for example, manufacture of electrical and electronic devices, preparation of thin films, high surface area catalysts in various chemical processes, application in cancer cells treatment, additives in germicides formulation, antibacterial materials [12, 13], additive for lubricating oils to improve antiwear properties caused by friction, and conductive inks and coatings [14].

Several synthetic methods for the preparation of copper nanoparticles have been developed including thermal and sonochemical reduction [15], metal vapor synthesis (MVS) [16], electron beam irradiation [17], microwave irradiation [18], reverse micelles [19], pulsed laser ablation [20], chemical reduction in aqueous solution [21], and polyol process [22]. Most of the preparation methods have several factors that limit their use in the synthesis of metal particles. For instance, the method of metal evaporation in gas phase has a high cost of starting materials and uses highly sophisticated equipment [6]; meanwhile the synthetic route by pulsed laser ablation is not a simple process, as it is inflexible and relatively costly [20]. Among these synthetic strategies, the wet chemical reduction is one of the most convenient methods because 
it has unique advantages over the other methods due to its versatility, since water has the ability to solubilize a wide variety of metallic salts, wherein the nanoparticles obtained by this method have well defined shapes and sizes [3]. Compared to conventional methods, products are cheaper and have higher purity [23], simple handling equipment is used and completion time of synthesis is short, and it is easy to scale up at an industrial level [6]. The reducing agents commonly used in the wet chemical reduction synthesis include formaldehyde [24], ascorbic acid [25], sodium hypophosphite [26], potassium borohydride and sodium [6, 27], and hydrazine hydrate [28]. However, some reducing agents are toxic and expensive, many of which have poor reductive capacity and can also introduce impurities to the process [6].

Unlike noble metals such as gold $(\mathrm{Au})$ and silver $(\mathrm{Ag})$, copper metal particles can not be obtained from the simple reduction of precursor salts in aqueous solution, since cupric oxide $(\mathrm{CuO})$ and cuprous oxide $\left(\mathrm{Cu}_{2} \mathrm{O}\right)$ could be generated during the process due to the presence of a large amount of water molecules. To protect copper nanoparticles against chemical oxidation during preparation and storage, sometimes the synthesis takes place in a nonaqueous medium using a low concentration of precursor and generally under inert atmospheres of Ar or $\mathrm{N}_{2}$ [29]. Occasionally, the above is not sufficient to prevent the oxidation of copper nanoparticles since their surface is very reactive. This makes necessary the use of stabilizers or agents such as water-soluble coating polymers, surfactants, or coordination ligands that have functional groups that can form complexes with copper ions present in the medium [3]. Compounds which are frequently used to meet the above include surfactants tetraethylenepentamine [30], cetyltrimethylammonium bromide (CTAB) [31], tetraoctylammonium bromide [32], and polyelectrolytes such as polyethyleneimine (PEI) [33], polyethylene glycols (PEG) [34], polyvinylpyrrolidone (PVP) [35], polyetheretherketone (PEEK) [36], and polyallylamine (PAAm) [37]. These materials can act as surface protectors, have the function of controlling size, size distribution, and shape of particles, and also prevent agglomeration thereof.

Here, we report the synthesis of copper nanoparticles using hydrazine hydrate $\left(\mathrm{N}_{2} \mathrm{H}_{4} \cdot \mathrm{H}_{2} \mathrm{O}\right)$ as a reducing agent, allylamine (AAm), linear polyallylamine (PAAm), and partially crosslinked polyallylamine (PAAmc) as stabilizing nitrogen agents. The synthesis was performed using different ratios of nitrogen ligands and using a constant concentration of $\mathrm{NaOH}$ and $\mathrm{N}_{2} \mathrm{H}_{4} \cdot \mathrm{H}_{2} \mathrm{O}$.

\section{Experimental}

2.1. Materials and Reagents. Copper(II) sulfate pentahydrate $\left(\mathrm{CuSO}_{4} \cdot 5 \mathrm{H}_{2} \mathrm{O}\right)$, hydrazine hydrate solution (50-60\%), poly(allylamine) solution (PAAm) (Mw $17000,20 \mathrm{wt} \%$ aqueous solution), and sodium hydroxide (98.8\%) were obtained from Sigma-Aldrich and were used without previous purification. PAAmc was obtained by PAAm's warming at $40^{\circ} \mathrm{C}$ for two months. The moles of PAAm were calculated by the molecular weight of the repeating unit $\left[\mathrm{CH}_{2}-\mathrm{CH}\left(\mathrm{CH}_{2} \mathrm{NH}_{2}\right)\right]$ of $57 \mathrm{~g} / \mathrm{mol}$. Different molar ratios of nitrogen ligands $/ \mathrm{CuSO}_{4} \cdot 5 \mathrm{H}_{2} \mathrm{O}$ were used. The reactions with
PAAmc used higher $\mathrm{H}_{2} \mathrm{O} / \mathrm{CuSO}_{4} \cdot 5 \mathrm{H}_{2} \mathrm{O}$ molar ratios due to the lower solubility of the ligand in $\mathrm{H}_{2} \mathrm{O}$.

2.2. Synthesis of Copper Nanoparticles. The synthesis of copper nanoparticles was performed using wet chemical reduction in atmospheric air; the following procedure describes the synthesis of NPs R4. In a flat bottom flask $\mathrm{CuSO}_{4} \cdot 5 \mathrm{H}_{2} \mathrm{O}$ $(0.5 \mathrm{~g}, 2.0 \mathrm{mmol})$ was added, which was dissolved in $30 \mathrm{~mL}$ of distilled water under magnetic stirring for 10 minutes at $60^{\circ} \mathrm{C}$. Then $0.065 \mathrm{~g}$ of PAAm was dissolved in $10 \mathrm{~mL}$ of distilled water and then added in a dropwise manner and stirred for 10 minutes. Subsequently, $33 \mathrm{~mL}$ of $\mathrm{NaOH}(0.5 \mathrm{M})$ was added dropwise and allowed to react for 30 minutes followed by the addition of $2.4 \mathrm{~mL}(76.9 \mathrm{mmol})$ of hydrazine. The reaction mixture was maintained at $60^{\circ} \mathrm{C}$ with constant stirring. The nanoparticles were recovered by centrifugation at $15,000 \mathrm{rpm}$ and $25^{\circ} \mathrm{C}$; solids were washed two times with distilled water and one time with ethanol. Final product was dried at $60^{\circ} \mathrm{C}$ for 2 hours; the NPs R4 were obtained as a black powder (0.0952 g, 75\%).

The yields were determined by the following equation:

$$
\text { Yield } \%=\left[\frac{\text { Experimental yield }}{\text { Theoretical yield }}\right] \times 100,
$$

theoretical yield $=\left[\left(\mathrm{g}, \mathrm{CuSO}_{4} \cdot 5 \mathrm{H}_{2} \mathrm{O}\right) /(\right.$ Molecular weight, $\left.\left.\mathrm{CuSO}_{4} \cdot 5 \mathrm{H}_{2} \mathrm{O}\right)\right] \times[(63.5 \mathrm{~g} \mathrm{Cu}) /(1 \mathrm{~mol} \mathrm{Cu})]$, experimental yield $=(\mathrm{g}$, Sample R1-R4) $(\% \mathrm{Cu}) / 100 ; \% \mathrm{Cu}=100-\%$ loss mass at $\leq 600^{\circ} \mathrm{C}$ (Figure 6).

2.3. Characterization Techniques. X-ray diffraction (XRD) technique was employed in order to know the oxidation state and chemical stability of the synthesized copper nanoparticles on a Siemens D-5000 diffractometer with a scanning interval in the $2 \theta$ range of 30 to $80^{\circ}$ with a scan speed of $0.02^{\circ}$ s; the radiation employed was copper $\mathrm{K} \alpha$ with a wavelength of $1.54056 \AA$; values of $25 \mathrm{~mA}$ and $35 \mathrm{kV}$ were used for intensity and voltage, respectively. The average particle diameter was determined from the XRD diffractograms of each sample based on the Debye-Scherrer equation taking the most intense peak for calculation $[38,39]$. Transmission electron microscopy (TEM) was used in order to observe the morphology and particle size distribution of the copper samples on a FEI Titan high-resolution electron microscope operating at $300 \mathrm{kV}$.

Thermogravimetric analysis (TGA) was employed to analyze the thermal behavior of $\mathrm{Cu}$ particles and the thermal stability of coating using a DuPont Instruments 951 analyzer. Operating conditions were a heating rate of $10^{\circ} \mathrm{C} / \mathrm{min}$. and air atmosphere with a gas flow of $50 \mathrm{~mL} / \mathrm{min}$. The runs of the samples were carried out from $30^{\circ} \mathrm{C}$ to $600^{\circ} \mathrm{C}$ in $\mathrm{N}_{2}$ atmosphere. Once $600^{\circ} \mathrm{C}$ was reached $\mathrm{N}_{2}$ atmosphere was changed for $\mathrm{O}_{2}$.

Differential scanning calorimetry analyses (DSC) were performed on a modulated TA Instrument 2920, at a heating rate of $10^{\circ} \mathrm{C} / \mathrm{min}$ on a temperature range from -40 to $160^{\circ} \mathrm{C}$. Through a second heating cycle the thermal and mechanical history of the samples was eliminated. 
TABLE 1: Results of the synthesis of copper nanoparticles by wet chemical reduction method.

\begin{tabular}{lcccccc}
\hline Sample & PAAm $/ \mathrm{Cu}$ & $\mathrm{AAm} / \mathrm{Cu}$ & Chemical composition & Average diameter $(\mathrm{nm})$ & Yield (\%) & Ligand (\%) \\
\hline $\mathrm{R}^{*}$ & 2.00 & 0.0 & $\mathrm{Cu}$ & 13.0 & 30.0 & 52.0 \\
$\mathrm{R} 2$ & 0.0 & 97.0 & $\mathrm{Cu}$ & 24.0 & 36.0 & 5.3 \\
$\mathrm{R} 3$ & 0.11 & 0.0 & $\mathrm{Cu}$ & 19.0 & 86.0 & 3.2 \\
$\mathrm{R} 4$ & 0.46 & 0.0 & $\mathrm{Cu}$ & 5.1 \\
\hline
\end{tabular}

Molar ratios of reagents in the synthesis of copper nanoparticles using a $\mathrm{N}_{2} \mathrm{H}_{4} / \mathrm{Cu}$ molar ratio of 38.0 and a NaOH/Cu molar ratio of 8.0. ${ }^{*}$ Partially crosslinked PAAm.

\section{Results and Discussion}

3.1. Effect of Nitrogen Ligands PAAm and AAm on the Synthesis of Copper Nanoparticles. Table 1 presents the results obtained in the synthesis of nanoparticles obtained by wet chemical reduction method and Figure 1 shows the XRD diffractograms of copper nanoparticles identified as R1, R3, and R4 and synthesized under different PAAm/Cu molar ratios: 2.00, 0.11 , and 0.46 , respectively. Diffractogram of nanoparticles obtained using AAm (R2) is also shown. In Figure 1, it can be seen that all particles synthesized using nitrogen ligands exhibit three reflections located at $2 \theta=43.4,50.5$, and $74.0^{\circ}$, attributed to the (111), (200), and (220) crystal planes, respectively, belonging to pure copper with facecentered cubic symmetry (FCC) $[40,41]$ and corresponding to the diffraction pattern of metallic copper (JCPDS number 04-0836) [42], as shown at the bottom of this figure. In the case of diffractogram R1, besides the signals described above, some small signs located at $2 \theta=35.9$ and $38.6^{\circ}$ (shown in the black dashed circle on the diffractogram) are presented; this suggests the start of an oxidative process. The yields obtained when PAAm was employed as a ligand, R3 and R4, were 86\% and $75 \%$, respectively. The use of PAAmc (R1) and AAm (R2) led to lower yields of $52 \%$ and $36 \%$, respectively. The percentage of ligand or particle coating was higher when using PAAmc (30.3\%). The use of PAAm and AAm led to low percentage of coating and the use of AAm ligand led to a higher percentage of nanoparticle coating despite having a much lower molecular weight than linear PAAm (see Table 1).

The particle diameter $(D)$ for the indicated samples was determined by the Debye-Scherrer equation $[38,39]$ as follows:

$$
D=\frac{0.94 \lambda}{\beta \cos \theta},
$$

where $\lambda$ is the copper wavelength (1.54056 $\AA$ ), $\theta$ is the Bragg diffraction angle, and $\beta$ is the half-width of the most intense diffraction peak. The average diameter calculated for the synthesized nanoparticles is shown in Table 1. Particles obtained with AAm (R2) showed the larger particle diameter of $30 \mathrm{~nm}$; this suggests that polymeric ligands promote the formation of small particles and is more evident when using PAAmc (R1), as the average diameter of these particles was $13 \mathrm{~nm}$.

In order to observe the morphology and particle size distribution each sample was analyzed by TEM. Figure 2 shows a TEM micrograph of particles R1. The presence of monodispersed spherical particles embedded within the matrix of

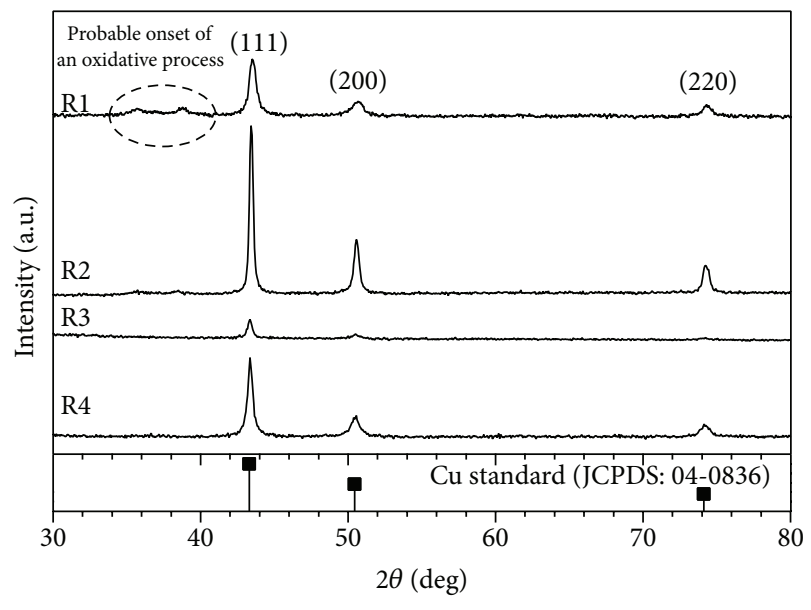

FIGURE 1: XRD diffractograms of copper nanoparticles synthesized by chemical reduction with different PAAm or $\mathrm{AAm} / \mathrm{Cu}$ molar ratios.

polymeric ligand PAAmc was observed. The particle size distribution of this sample based on the count of $\sim 220$ particles is shown at the upper left side of the histogram in Figure 2. In this figure, a size distribution was observed with relatively close interval sizes ranking between 0.5 and $11 \mathrm{~nm}$ with an average diameter of $3.9 \mathrm{~nm}$, which was slightly lower than the one calculated with the X-rays through the Debye-Scherrer equation with a value of $13.0 \mathrm{~nm}$ (see Table 1).

The synthesis of nanoparticles using AAm, identified as R2, was also analyzed by TEM (Figure 3 ). This micrograph illustrates a set of nanoparticles embedded in a mass of nitrogen ligand; its appearance is very similar to the coating present in the micrograph of Figure 2, corresponding to PAAmc. The particles, which form part of the polymer mass, present spherical and semispherical morphologies and are relatively scattered. According to the histogram of particle size distribution shown in Figure 3, a closed behavior is experimented with sizes within the range of 1 to $19 \mathrm{~nm}$ with an average diameter of $6.0 \mathrm{~nm}$. TEM analysis confirmed that the AAm ligand used in the synthesis of particles polymerized during the oxidation and reduction processes of the reaction. The coating of nanoparticles (Figure 3) is very similar to that observed coating when PAAmc was used (Figure 2); this suggests that the PAAm synthesized in situ may also have a certain degree of crosslinking. As previously mentioned, TGA analysis also gave evidence of the presence of PAAm as a coating in particles R2. In order to obtain further evidence, 


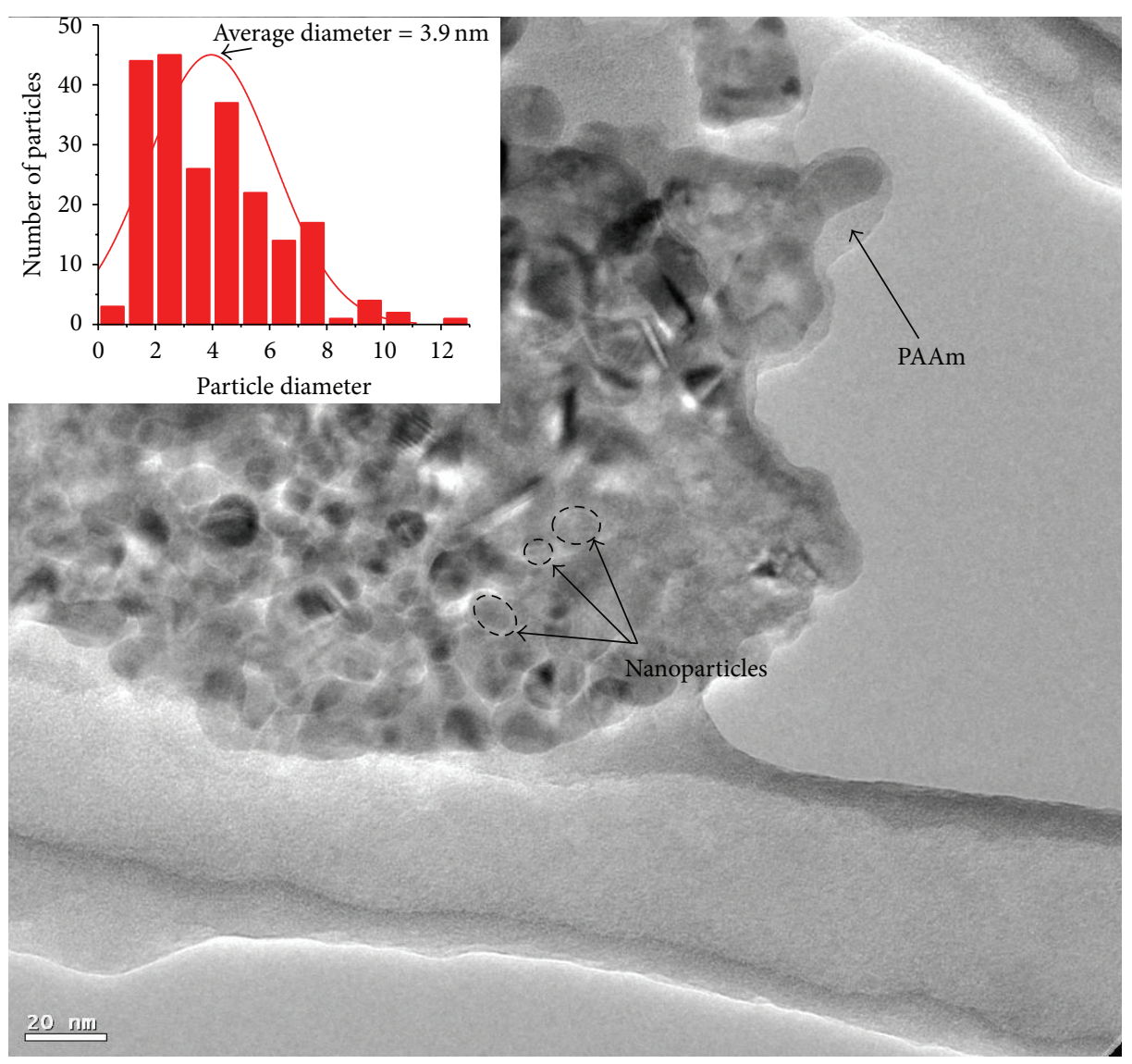

FIGURE 2: TEM micrograph of copper nanoparticles R1 synthesized by chemical reduction with a PAAm/Cu molar ratio of 2.00 .

the particles were analyzed by differential scanning calorimetry (DSC). The thermogram presented a transition glass at $25^{\circ} \mathrm{C}$, which may correspond to PAAm ligand. This value is higher than that reported in PAAm $\left(T_{g}=-6^{\circ} \mathrm{C}\right)$ and is explained by the coordination of amino groups to copper NPs and possible crosslinking of PAAm.

Particles synthesized with a PAAm/Cu molar ratio of 0.11 and labeled as R3 are shown in the TEM micrograph of Figure 4. In this micrograph, the following can be observed: individual copper particles with sizes in the range of 20 to $110 \mathrm{~nm}$ approximately and hemispherical and hexagonal morphologies, as well as polycrystalline aggregates of nanoparticles with sizes between 100 and $200 \mathrm{~nm}$ and subparticles with diameters of approximately 20 to $50 \mathrm{~nm}$ and hemispherical shapes. The particle size obtained by the Debye-Scherrer equation for this sample was $24 \mathrm{~nm}$ (see Table 1), which lies within the range measured by TEM.

Figure 5 shows a TEM micrograph of the particles synthesized with a PAAm/Cu molar ratio of 0.46 and identified as R4. The micrograph shows (bottom left side) isolated particles with sizes between 20 and $30 \mathrm{~nm}$, where it can be noticed that these particles agglomerate forming larger particles with hemispheric morphologies. The presence of subparticles can also be observed (pointed arrow) and the histogram shows the particle size distribution with an average diameter of $55 \mathrm{~nm}$ (upper left side). The particle size determined by the
Debye-Scherrer equation was $19.0 \mathrm{~nm}$ (Table 1) and is within the range observed by TEM. The black arrow points out the enlargement of a polycrystalline aggregate of nanoparticles whose surface is wrapped by a thin layer of PAAm with a thickness of about $5 \mathrm{~nm}$, acting as a surface coating.

Determination of average particle diameter by XRD and TEM leads to different results. The differences can be explained in terms of (1) morphologies and sizes of the agglomerates, (2) basic operating principles of the instruments, (3) sample preparation of each analysis, and (4) limitations of each instrument [43]. When the particles are delineated by a well defined boundary and the particle size distribution is narrow, a good correspondence between XRD and TEM is observed.

The weight percentages of copper nanoparticles, ligand, and volatiles were determined by TGA analysis in each of the particles synthesized R1-R4 (Figure 6). The results of the thermograms are shown in Table 2. The maximum degradation temperatures of R1-R4 were determined by the first derivative TGA (dTGA) curves (not shown in Figure 6) and were compared with the neat PAAm thermogram.

The thermogram of neat PAAm shows three weight-loss events; the first event is located in a temperature range of $\sim 30$ to $200^{\circ} \mathrm{C}$ and is attributed to water evaporation. The second loss is between temperatures of $\sim 200$ and $494^{\circ} \mathrm{C}$; the temperature where significant weight loss of ligand is most apparent 


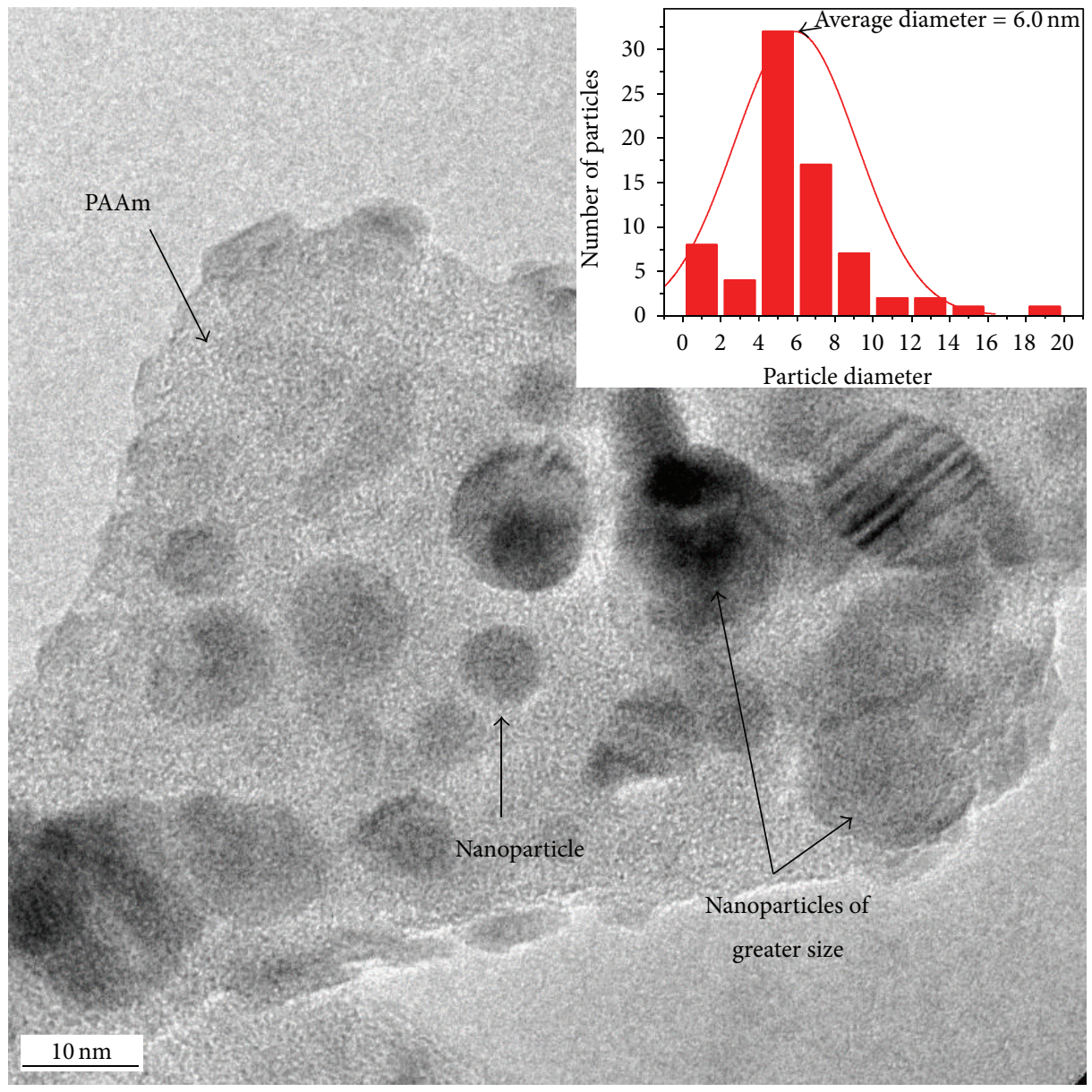

FIGURE 3: TEM micrograph of copper nanoparticles R2 synthesized by chemical reduction with a AAm/Cu molar ratio of 97.0.

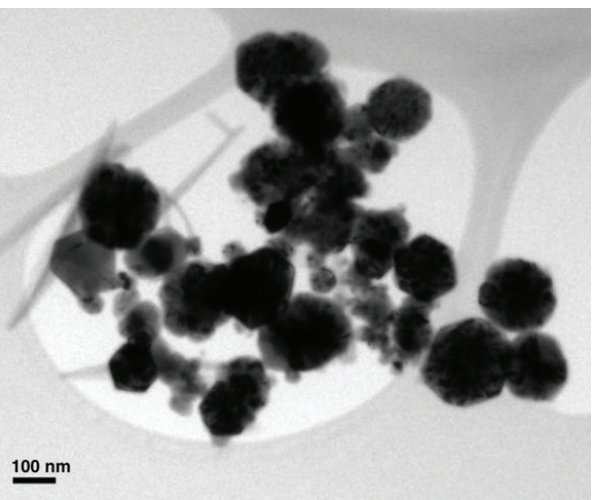

FIGURE 4: TEM micrograph of copper nanoparticles R3 synthesized by chemical reduction with a PAAm/Cu molar ratio of 0.11 .

is known as maximum degradation temperature and in the case of PAAm was $440^{\circ} \mathrm{C}$. This value is very close to that reported in the literature $[44,45]$. The third and final stage of material loss is between $600^{\circ} \mathrm{C}$ and $620^{\circ} \mathrm{C}$ and is assigned to the degradation of pyrolytic carbon from the polymer ligand due to the entrance of an oxygen source. The formation and thermal degradation of this type of coal have been reported in previous work [46].

The thermograms of R1-R4 presented a weight-loss pattern similar to that observed in neat PAAm. From $30^{\circ} \mathrm{C}$ to $200^{\circ} \mathrm{C}$ a weight loss assigned to volatile water and ethanol can be appreciated (Figure 6). In the case of particles R1 the value was $9.5 \%$ and was greater than the value presented in particles R2-R4 (Table 2); this is attributed to the higher solvent retention of partially crosslinked PAAm. The second loss of organic material occurs between 200 and $600^{\circ} \mathrm{C}$ and is assigned to the degradation of nitrogen ligand. The peak decomposition temperature of particles $\mathrm{R} 1$ is very similar to that presented by neat PAAm; a similar situation is observed in R3 and R4. Nanoparticles R2 presented a peak decomposition temperature of $354^{\circ} \mathrm{C}$; this temperature is $86^{\circ} \mathrm{C}$ lower than that observed in all the samples where PAAm was used for the synthesis; this suggests that during the synthesis of particles R2 the AAm ligand polymerized forming PAAm with low molecular weight and probably with a different structure to the linear. After $600^{\circ} \mathrm{C} \mathrm{R} 1$ presented a similar behavior to PAAm while particles R2-R4 showed a weight gain; this is attributed to the oxidation of copper metal caused by the flow of oxygen to which it was subjected. 


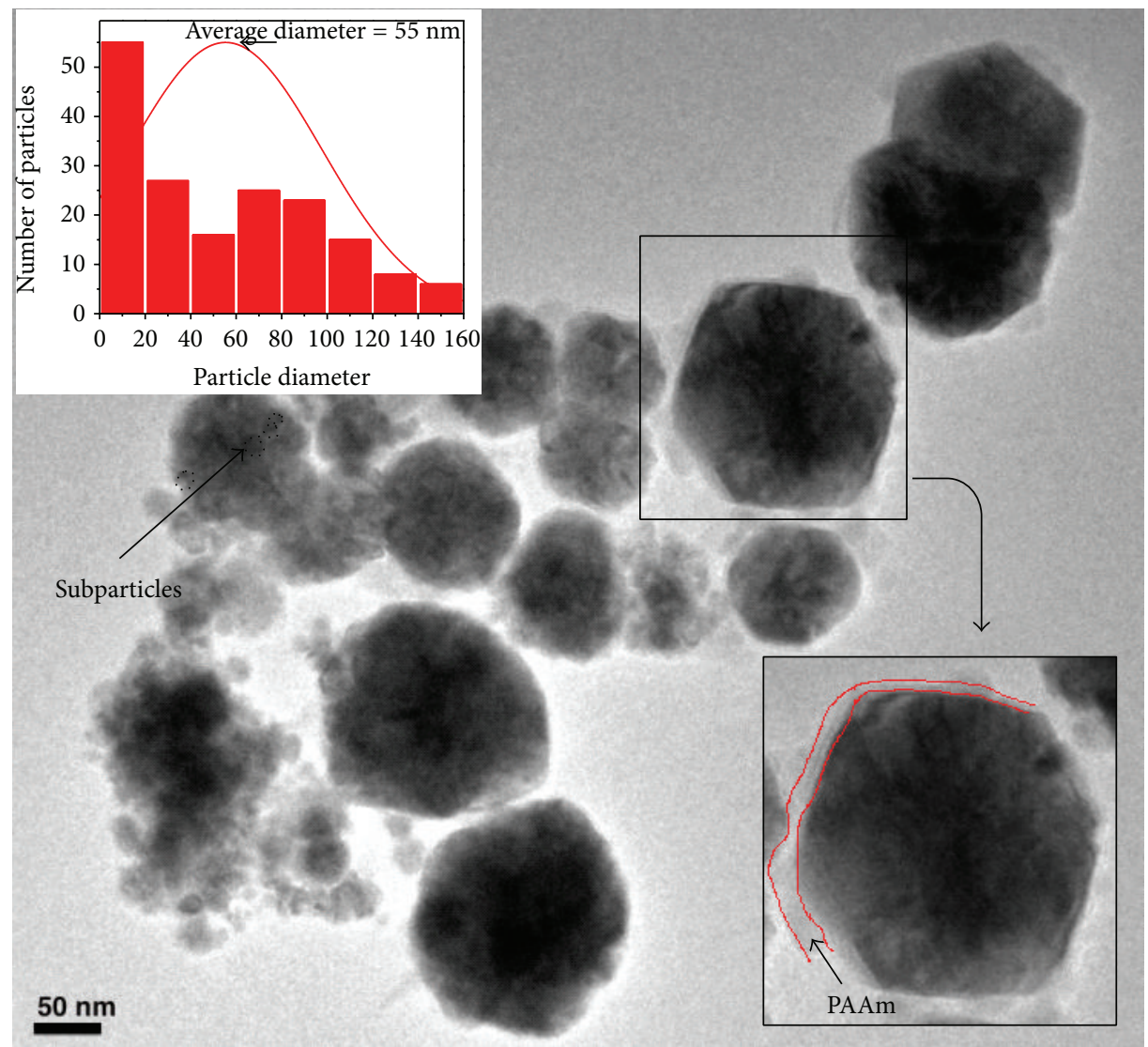

FIGURE 5: TEM micrograph of copper nanoparticles R4 synthesized through chemical reduction with a PAAm/Cu molar ratio of 0.46 .

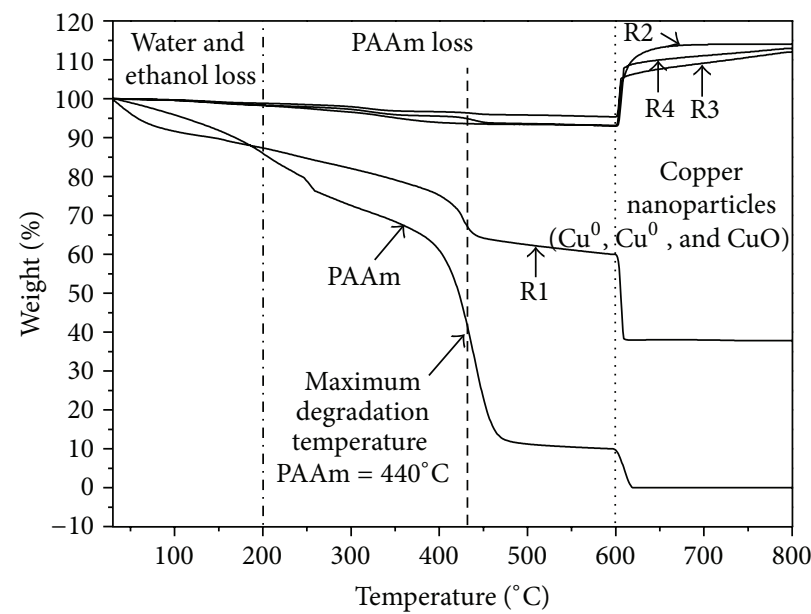

FIGURE 6: TGA thermograms of copper nanoparticles synthesized through chemical reduction with different PAAm or AAm/Cu molar ratios.

TABLE 2: TGA results of copper nanoparticles obtained with ligands PAAm and AAm.

\begin{tabular}{lccccc}
\hline Sample & PAAm $/ \mathrm{Cu}$ & $\mathrm{Cu}(\%)^{* *}$ & Ligand $(\%)$ & Solvent $(\%)$ & Maximum degradation temperature $\left({ }^{\circ} \mathrm{C}\right)$ \\
\hline PAAm & 0.0 & 0.0 & 86.0 & 14.0 & $\sim 440.0$ \\
R1 & 2.00 & 60.2 & 30.3 & 9.5 & $\sim 428.0$ \\
R2 & 0.0 & 93.2 & 5.6 & 1.2 & $\sim 354.0$ \\
R3 & 0.11 & 95.4 & 3.2 & 1.4 & $\sim 440.3$ \\
R4 & 0.46 & 93.1 & 5.1 & 1.8 & $\sim 441.1$ \\
\hline
\end{tabular}

${ }^{*}$ Obtained with AAm ligand, ${ }^{* *} \% \mathrm{Cu}=100-\%$ loss mass at $\leq 600^{\circ} \mathrm{C}$. 
The $\mathrm{Cu}$ percentage in nanoparticles $\mathrm{R} 2-\mathrm{R} 4$ was greater than $90 \%$ while for $\mathrm{R} 1$ it was $60.2 \%$. This value is attributed to the lower coordination ability of partially crosslinked PAAm used during the synthesis. Particles R1 presented 30.3\% of nitrogen ligand, a higher value than R2-R4 (Table 2). It is clear that crosslinking reduces the coordination of the amino groups but promotes retention of water and ethanol.

The use of AAm ligand during the synthesis of nanoparticles $\mathrm{R} 2$ could be a good alternative to reduce the costs of the synthetic method due to the fact that the price of AAm is less than PAAm. Although the yield in R2 was low, the optimization of the synthesis using AAm could be of academic and industrial interest. In addition, the synthesis of nanoparticles in the presence of a mixture of PAAm and AAm could be of interest. These studies are currently underway in our group and will be published in the near future.

\section{Conclusions}

Synthesis of metallic copper particles was achieved using nitrogen ligands AAm and PAAm, leading to metallic copper nanoparticles free of oxidized copper nanoparticles. The average particle diameters determined by XRD were lower than $31 \mathrm{~nm}$. The use of PAAm promotes the synthesis of particles with low percentage of coating and high yields, while the use of partially crosslinked PAAm favours the preparation of nanoparticles with high percentage of coating and moderate yields. The use of AAm in the synthesis of copper nanoparticles does not have a significant effect on the particle size; however, it may affect the yield. The TGA, DSC, and TEM analyses of nanoparticles R2 using AAm suggest the in situ formation of PAAm.

\section{Conflict of Interests}

The authors declare that there is no conflict of interests regarding the publication of this paper.

\section{Acknowledgments}

The authors gratefully acknowledge the financial support of CONACYT Grant 127151/EU 7th FP Grant 26396, CONACYT Grant 132578, and CuVito Project. R. Sierra-Avila wishes to thank CONACYT for the Scholarship no. 23415. Finally, the authors would like to thank V. Comparán-Padilla and G. Mendez-Padilla for their valuable technical support.

\section{References}

[1] P. Kanninen, C. Johans, J. Merta, and K. Kontturi, "Influence of ligand structure on the stability and oxidation of copper nanoparticles," Journal of Colloid and Interface Science, vol. 318, no. 1, pp. 88-95, 2008.

[2] X. Su, J. Zhao, H. Bala et al., "Fast synthesis of stable cubic copper nanocages in the aqueous phase," Journal of Physical Chemistry C, vol. 111, no. 40, pp. 14689-14693, 2007.

[3] J. Yang, S. Yang, T. Okamoto et al., "Synthesis of copper monolayer and particles at aqueous-organic interface," Surface Science, vol. 600, no. 24, pp. L318-L320, 2006.
[4] K. Judai, S. Numao, J. Nishijo, and N. Nishi, "In situ preparation and catalytic activation of copper nanoparticles from acetylide molecules," Journal of Molecular Catalysis A: Chemical, vol. 347, no. 1-2, pp. 28-33, 2011.

[5] T. M. D. Dang, T. T. T. Le, E. Fribourg-Blanc, and M. C. Dang, "Synthesis and optical properties of copper nanoparticles prepared by a chemical reduction method," Advances in Natural Sciences: Nanoscience and Nanotechnology, vol. 2, no. 1, Article ID 015009, 2011.

[6] Q. L. Zhang, Z. M. Yang, B. J. Ding, X. Z. Lan, and Y. J. Guo, "Preparation of copper nanoparticles by chemical reduction method using potassium borohydride," Transactions of Nonferrous Metals Society of China, vol. 20, supplement 1, pp. s240s244, 2010.

[7] G. H. Chan, J. Zhao, E. M. Hicks, G. C. Schatz, and R. P. van Duyne, "Plasmonic properties of copper nanoparticles fabricated by nanosphere lithography," Nano Letters, vol. 7, no. 7, pp. 1947-1952, 2007.

[8] W. Yu, H. Xie, L. Chen, and Y. Li, "Investigation on the thermal transport properties of ethylene glycol-based nanofluids containing copper nanoparticles," Powder Technology, vol. 197, no. 3, pp. 218-221, 2010.

[9] A. Ghasemi, E. Ghasemi, and E. Paimozd, "Influence of copper cations on the magnetic properties of NiCuZn ferrite nanoparticles," Journal of Magnetism and Magnetic Materials, vol. 323, no. 11, pp. 1541-1545, 2011.

[10] J. Ramyadevi, K. Jeyasubramanian, A. Marikani, G. Rajakumar, and A. A. Rahuman, "Synthesis and antimicrobial activity of copper nanoparticles," Materials Letters, vol. 71, pp. 114-116, 2012.

[11] T. Theivasanthi and M. Alagar, "Studies of copper nanoparticles effects on micro-organisms," Annals of Biological Research, vol. 2, no. 3, pp. 368-373, 2011.

[12] M. H. Habibi, R. Kamrani, and R. Mokhtari, "Fabrication and characterization of copper nanoparticles using thermal reduction: the effect of nonionic surfactants on size and yield of nanoparticles," Microchimica Acta, vol. 171, no. 1-2, pp. 91-95, 2010.

[13] F. Rispoli, A. Angelov, D. Badia, A. Kumar, S. Seal, and V. Shah, "Understanding the toxicity of aggregated zero valent copper nanoparticles against Escherichia coli," Journal of Hazardous Materials, vol. 180, no. 1-3, pp. 212-216, 2010.

[14] B. K. Park, D. Kim, S. Jeong, J. Moon, and J. S. Kim, "Direct writing of copper conductive patterns by ink-jet printing," Thin Solid Films, vol. 515, no. 19, pp. 7706-7711, 2007.

[15] N. A. Dhas, C. P. Raj, and A. Gedanken, "Synthesis, characterization, and properties of metallic copper nanoparticles," Chemistry of Materials, vol. 10, no. 5, pp. 1446-1452, 1998.

[16] A. A. Ponce and K. J. Klabunde, "Chemical and catalytic activity of copper nanoparticles prepared via metal vapor synthesis," Journal of Molecular Catalysis A: Chemical, vol. 225, no. 1, pp. $1-6,2005$.

[17] R. Zhou, X. Wu, X. Hao, F. Zhou, H. Li, and W. Rao, "Influences of surfactants on the preparation of copper nanoparticles by electron beam irradiation," Nuclear Instruments and Methods in Physics Research B: Beam Interactions with Materials and Atoms, vol. 266, no. 4, pp. 599-603, 2008.

[18] H. Zhu, C. Zhang, and Y. Yin, "Rapid synthesis of copper nanoparticles by sodium hypophosphite reduction in ethylene glycol under microwave irradiation," Journal of Crystal Growth, vol. 270 , no. 3-4, pp. 722-728, 2004. 
[19] I. Lisiecki and M. P. Pileni, "Synthesis of copper metallic clusters using reverse micelles as microreactors," Journal of the American Chemical Society, vol. 115, no. 10, pp. 3887-3896, 1993.

[20] R. M. Tilaki, A. Iraji Zad, and S. M. Mahdavi, "Size, composition and optical properties of copper nanoparticles prepared by laser ablation in liquids," Applied Physics A, vol. 88, no. 2, pp. 415-419, 2007.

[21] L. Quoc, J. Hwa, C. Woo et al., "Copper nanoparticles incorporated with conducting polymer: effects of copper concentration and surfactants on the stability and conductivity," Journal of Colloid and Interface Science, vol. 365, no. 1, pp. 103-109, 2012.

[22] B. K. Park, S. Jeong, D. Kim, J. Moon, S. Lim, and J. S. Kim, "Synthesis and size control of monodisperse copper nanoparticles by polyol method," Journal of Colloid and Interface Science, vol. 311, no. 2, pp. 417-424, 2007.

[23] T. M. D. Dang, T. T. T. Le, E. Fribourg-Blanc, and M. C. Dang, "The influence of solvents and surfactants on the preparation of copper nanoparticles by a chemical reduction method," Advances in Natural Sciences: Nanoscience and Nanotechnology, vol. 2, no. 2, Article ID 025004, 2011.

[24] R. Liao, B. Sun, and H. B. Tan, "Research of preparation of ultrafine copper powder using formaldehyde as reductive agent," Journal of Chengdu University of Technology, vol. 30, no. 4, pp. 417-421, 2003.

[25] Q. Zhou, P. Jiang, W. Zhu, and D. Zhao, "Preparation and characterization of anti-oxidation copper nanopowders," Rare Metal Materials and Engineering, vol. 33, no. 2, pp. 179-182, 2004.

[26] Z. Zhang, X. Han, and M. Sun, "Preparation of nanocopper powder," Fine Chemical, vol. 17, no. 2, pp. 69-71, 2000.

[27] Q.-M. Liu, D.-B. Zhou, Y. Yamamoto, R. Ichino, and M. Okido, "Preparation of $\mathrm{Cu}$ nanoparticles with $\mathrm{NaBH}_{4}$ by aqueous reduction method," Transactions of Nonferrous Metals Society of China, vol. 22, no. 1, pp. 117-123, 2012.

[28] H. Chen, J. Tang, J. Xin, and W. Su, "Preparation of copper nanoparticles by reducing hydrazine," New Chemical Materials, vol. 33, no. 11, pp. 48-50, 2005.

[29] S. Qiu, J. Dong, and J. Chen, "Preparation of Cu nanoparticles from water-in-oil microemulsions," Journal of Colloid and Interface Science, vol. 216, no. 2, pp. 230-234, 1999.

[30] C. Wu, B. Mosher, and T. Zeng, "Simple one-step synthesis of uniform disperse copper nanoparticles," MRS Proceedings, vol. 879, 2005.

[31] B. Bozzini, L. Urzo, M. Re, and F. Riccardis, "Electrodeposition of $\mathrm{Cu}$ from acidic sulphate solutions containing cetyltrimethylammonium bromide (CTAB)," Journal of Applied Electrochemistry, vol. 38, no. 11, pp. 1561-1569, 2008.

[32] N. Dadgostar, S. Ferdous, and D. Henneke, "Colloidal synthesis of copper nanoparticles in a two-phase liquid-liquid system," Materials Letters, vol. 64, no. 1, pp. 45-48, 2010.

[33] P. Pulkkinen, J. Shan, K. Leppänen et al., "Poly(ethylene imine) and tetraethylenepentamine as protecting agents for metallic copper nanoparticles," ACS Applied Materials \& Interfaces, vol. 1, no. 2, pp. 519-525, 2009.

[34] X. Cheng, X. Zhang, H. Yin, A. Wang, and Y. Xu, "Modifier effects on chemical reduction synthesis of nanostructured copper," Applied Surface Science, vol. 253, no. 5, pp. 2727-2732, 2006.

[35] H. Huang, F. Yan, Y. Kek et al., "Synthesis, characterization, and nonlinear optical properties of copper nanoparticles," Langmuir, vol. 13, no. 2, pp. 172-175, 1997.
[36] F. Schwarz, G. Thorwarth, and B. Stritzker, "Synthesis of silver and copper nanoparticle containing a-C:Hby ion irradiation of polymers," Solid State Sciences, vol. 11, no. 10, pp. 1819-1823, 2009.

[37] Y. Wang and T. Asefa, "Poly(allylamine)-stabilized colloidal copper nanoparticles: synthesis, morphology, and their surfaceenhanced raman scattering properties," Langmuir, vol. 26, no. 10, pp. 7469-7474, 2010.

[38] J. I. Langford and A. J. C. Wilson, "Scherrer after sixty years: a survey and some new results in the determination of crystallite size," Journal of Applied Crystallography, vol. 11, no. 2, pp. 102113, 1978.

[39] A. Monshi, "Modified scherrer equation to estimate more accurately nano-crystallite size using XRD," World Journal of Nano Science and Engineering, vol. 2, no. 3, pp. 154-160, 2012.

[40] O. Mondal, A. Datta, D. Chakravorty, and M. Pal, "Ultrafine narrow dispersed copper nanoparticles synthesized by a facile chemical reduction method," MRS Communications, vol. 3, no. 2, pp. 91-95, 2013.

[41] S. V. Saikova, S. A. Vorob'ev, R. B. Nikolaeva, and Y. L. Mikhlin, "Conditions for the formation of copper nanoparticles by reduction of copper(II) ions with hydrazine hydrate solutions," Russian Journal of General Chemistry, vol. 80, no. 6, pp. 11221127, 2010.

[42] P. A. Swarthmore, Card. No 04-0836, JCPDS, International Center for Powder Difraction Data, 1989.

[43] H. Taib and C. C. Sorrell, "Assessment of particle sizing methods applied to agglomerated nanoscale tin oxide $\left(\mathrm{SnO}_{2}\right)$," Journal of the Australian Ceramic Society, vol. 44, no. 2, pp. 47-51, 2008.

[44] J. M. El Khoury, D. Caruntu, C. J. O’Connor, K.-U. Jeong, S. Z. D. Cheng, and J. Hu, "Poly(allylamine) stabilized iron oxide magnetic nanoparticles," Journal of Nanoparticle Research, vol. 9, no. 5, pp. 959-964, 2007.

[45] S. J. Kim, S. J. Park, M.-S. Shin, Y. H. Lee, N. G. Kim, and S. I. Kim, "Thermal characteristics of IPNs composed of polyallylamine and chitosan," Journal of Applied Polymer Science, vol. 85, no. 9, pp. 1956-1960, 2002.

[46] J. Rodriguez-Mirasol, T. Cordero, L. R. Radovic, and J. J. Rodriguez, "Structural and textural properties of pyrolytic carbon formed within a microporous zeolite template," Chemistry of Materials, vol. 10, no. 2, pp. 550-558, 1998. 

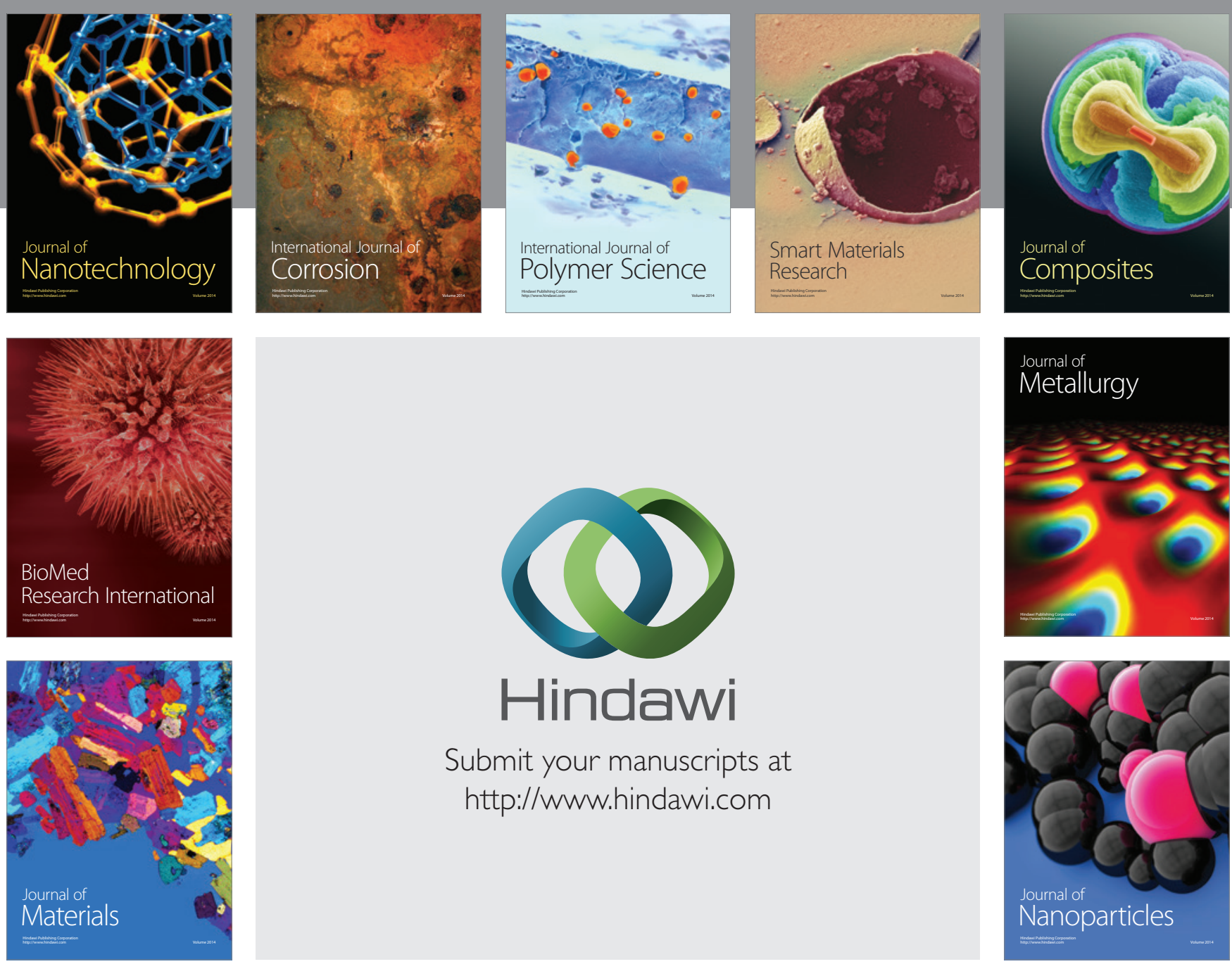

Submit your manuscripts at http://www.hindawi.com
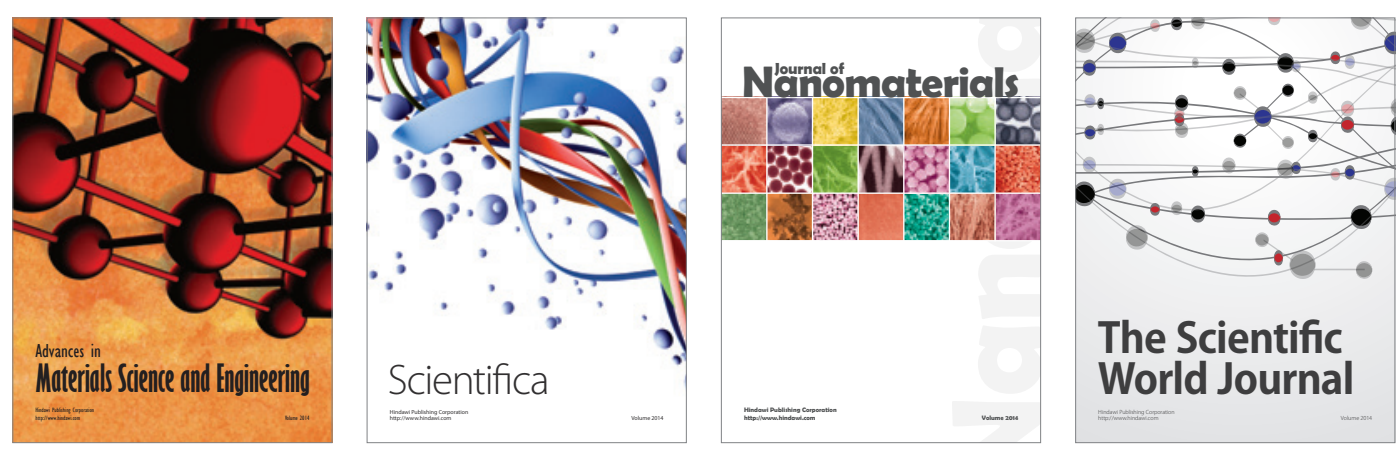

\section{The Scientific World Journal}
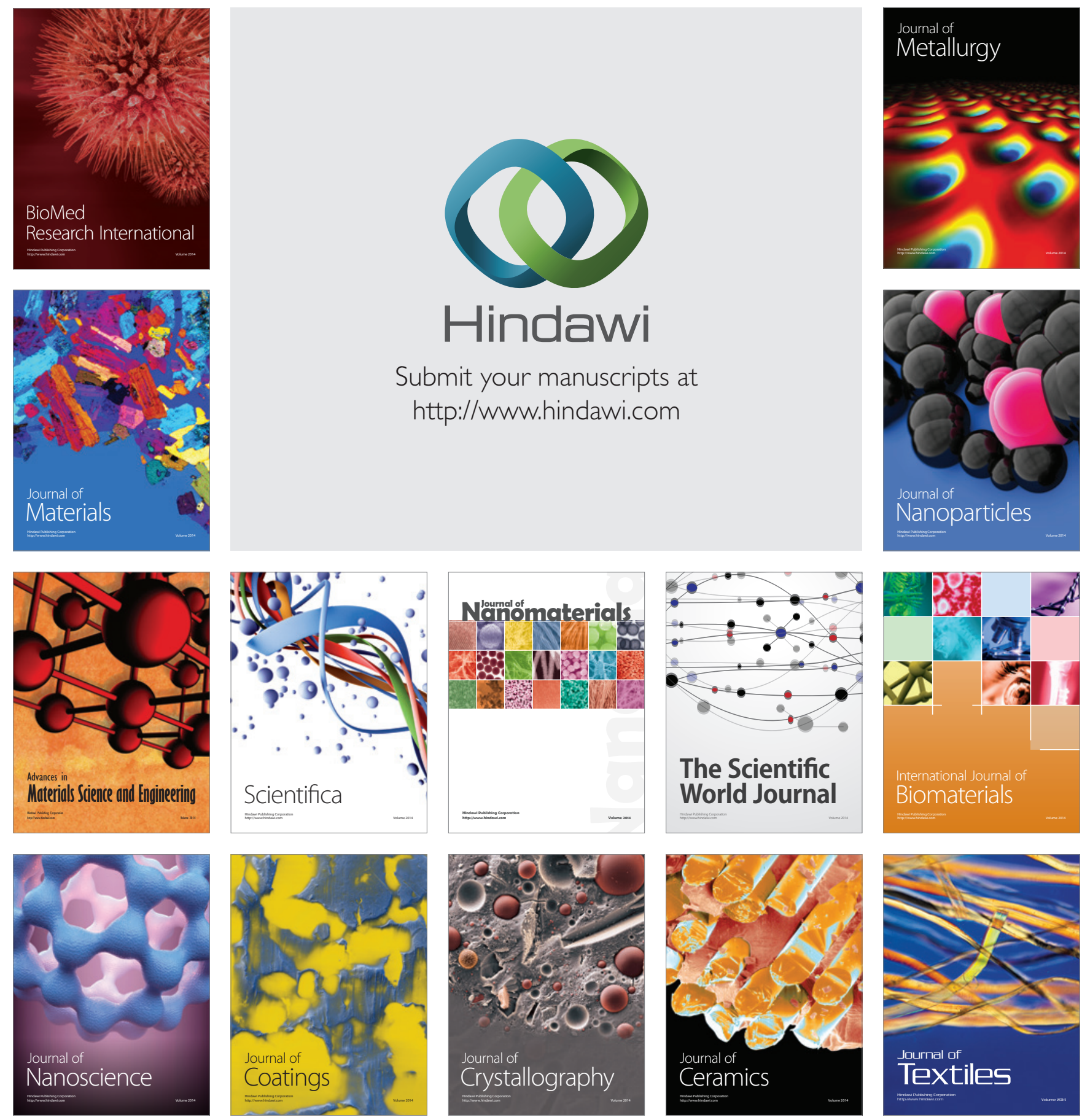